\title{
Le Paléolithique supérieur de plein air (sa recherche)
}

\author{
J. GAUSSEN
}

Il est des réalités que tout le monde connait mais dont personne ne tient compte. II en est ainsi pour la recherche en plein air. II ne s'agit pas ici de la recherche des truffes, de la nappe d'eau souterraine ou des métaux précieux mais tout simplement des vestiges archéologiques et, en particulier, du Paléolithique supérieur.

Chacun sait qu'il n'est pas facile de trouver une aiguille dans une botte de foin et cependant chacun se comporte comme si l'aiguille en question était d'une couleur flamboyante et placée, bien en évidence, sur la surface la plus en vue de la dite botte.

Quelques allées et venues dans un champ, un examen rapide des coupes de terrain voisines s'il y en a, un ou deux sondages pratiqués à la pioche et à la pelle quand ce n'est pas avec une sonde à prélèvement et l'endroit est désormais réputé vierge de tout vestige préhistorique. II est banal de lire dans les publications sur les stations dites de surface: «nous avons effectué un sondage en l'endroit le plus riche mais n'avons rien découvert". Par rien découvert il faut entendre évidemment la trouvaille de couches en place.

Et pourtant ces objets encore en place existent. Le problème est de les trouver. Revenant cinq ans après en un secteur des Vosges où en 1944 les munitions avaient été abondemment dépensées je n'ai pu remasser le moindre douille ni le moindre éclat d'obus. Le patron d'une scierie voisine me montra rageusement une boite pleine d'éclats: "les sapins ici en sont bourrés. Une lame de scie ne fait pas la semaine. Emportez donc la boite si elle vous fait plaisir".

Je n'avais pu découvrir le moindre éclat et pourtant il y en avait. 
Il en est ainsi pour les objets préhistoriques. Ils ne sont pas tous localisés dans les gisements sous abri ou dans les grandes stations de plein air encore bien rares à ce jour. Ils sont présents un peu partout, tout au moins dans les régions qui ont connu une activité humaine durant les millénaires du Paléolithique supérieur. A la cadence d'une cinquantaine d'installations, bivouacs, haltes de plus ou moins longue durée, lieux de dépeçage ou ateliers de débitage, par an et pour l'ensemble du Périgord cela représente près d'un million de points où les espoirs de découvertes ne sont pas tous nuls.

Pour quelles raisons certains secteurs sont ils considérés comme des No mans land préhistoriques alors que les motifs n'en sont pas évidents? Vient en premier l'absence totale des recherches. Bien des endroits en Périgord n'ont jamais été prospectés. Dans son inventaire des stations préhistoriques du Périgord, Denis Peyrony ajoute souvent la mention: "canton à prospecter soigneusement" (D. Peyrony, 1944). Le profane ramasse les bifaces, les pointes de flèche ou les haches polies. Seuls le préhistorien ou l'amateur compétent savent ce que représente cet éclat en apparence informe qu'est le burin sur coche latérale ou encore la pièce esquillée. Ces raisons la sont évidentes et il n'est pas besoin de les commenter longuement: on n'a pas trouvé de pétrole au Sahara avant que les géologues ne l'aient recherché.

Comment se pose le problème pour les régions prospectées? La plupart de ces prospections ou soi disant telles ne son en fait que des promenades ou des examens très rapides à des fins qui ne sont pas toujours d'ordre archéologique. Les découvertes ne sont alors possibles que dans la mesure ou les vestiges sont en surface, bien conservés ce qui n'est pas toujours le cas et surtout très abondants ce qui est encore plus rare.

Dans la vallée de l'Isle un seule station a été découverte de la sorte: Solvieux; mais il s'agit d'une station très vaste, elle a près de deux hectares de superficie et le matériel découvert en surface se chiffre par milliers de pièces (L. Peyrille, 1948; L. Peyrille et S. Blanc 1952). Ces énormes gisements ne sont pas très abondants et le plus souvent on a affaire à des vestiges dont la densité ne saurait se comparer en rien à celles des abris sous roche où le materiel est concentré sur des surfaces genéralement très restreintes. Les stations de plein air n'en sont pas plus pauvres pour autant. Prenons un exemple précis: le propriètaire d'un troupeau est il plus riche si ses bètes sont enfermées dans une étable ou si elles sont disséminées dans les champs. II n'empèche que l'acheteur sera plus intéréssé par l'étable. Qu'il s'agisse de matériel épars sur le sol ou d'une couche mise au jour lors de travaux de terrassement, seuls 
seront remarqués par le profane ou mème le préhistorien les dépots très apparents et par conséquent très riches. Les autres n'apparaissent que de manière fortuite ou au prix de recherches minutieuses et prolongées. Je me contenterai de citer deux exemples particulièrement instructifs. Celui de Guillassou tout d'abord.

La station de plein air de Guillassou occupe une superficie de près de deux hectares sur laquelle le matériel de surface est très abondant. Avec l'aide de J.C. Moissat nous pratiquons une série de sondages dont le nombre ne saurait étre précisé, peut ètre une douzaine, avant de découvrir un habitat magdalénien bien en place (J. Gaussen 1980).

Ceci se passait il a plus de vingt ans et pour ètre plus prècis dans les premiers jours du mois d'avril 1965. Au cours des fouilles qui vont durer pendant deux ans il est procédé à de nouveaux sondages. Ils sont tous négatifs. Trois ans plus tard le fond de cabane est remis en état pour être montré aux congressistes de l'INQUA. A cette occasion de nouvelles recherches sont entreprises avec l'aide de l'équipe américaine du Pr. Sackett. Pas un seul éclat en place n'est découvert.

Par la suite un chateau d'eau est construit sur le site. Rien n'apparait dans les coupes de terrain faites par le creusement des fondations et l'examen des déblais n'est guère encourageant. Les recherches s'arrèteraient la s'il n'était découvert de temps en temps, dans la terre fraichement labourée, des éclats porteurs de ces granulations d'oxydes métalliques que l'on ne trouve que sur les objets extraits récemment d'une couche en place. Pendant une quinzaine d'années les travaux agricoles sont suivis de maniére assez régulière et des explorations au stylet sonde effectués à chaque passage sur le terrain.

En 1980, profitant d'un changement de culture, je reprends les recherches et pratique un certain nombre de sondages - prés d'une dizaine- dans la partie est du champ. L'un d'eux découvre deux petits éclats apparemment bien en place. II n'y a rien aux alentours. Persuadé que le gisement est épuisé je cesse pendant plusieurs annés de m'intéresser à ce site qui n'apporte plus que des sujets de désillusion. Mais il ne faut jamais dire "fontaine je ne boirai plus de ton eau". Le 21 février 1988, averti par le propriétaire d'un changement prochain de culture, avec l'aide de J.C. Moissat je reprends les sondages de la dernière chance. Durant toute une matinée nous ne trouvons que des terrains bouleversés ou stériles mais dans l'aprés midi c'est la découverte d'un niveau intact renfermant près de 200 outils, en majorité des burins ( 80 pour 100) sur 3 à 4 métres carrés environ (J. Gaussen et J.C. Moissat, 1989). Nous sommes à une vingtaine de mètres de l'habitat fouillé en 1965 et en un 
endroit où de multiples recherches avient déja été effectuées. Je ne saurais préciser le nombre exact des sondages qui ont été pratiqués sur la station avant cette dernière découverte. II y en a eu certainement plus d'une centaine sans compter les innombrables investigations au stylet sonde. II est donc hasardeux d'affirmer sur la foi de deux ou trois trous qu'un site de plein air est vierge de tout vestige d'occupation humaine.

Le cas de Guillassou n'est pas unique. Les découvertes du Breuil, du Plateau Parrain, du Chatenet et du Mas de Sourzac ont été elles aussi le résultat de recherches prolongées.

Le deuxième exemple concerne l'examen des coupes de terrain. II semblerait de prime abord qu'il ne présente aucune espèce de difficulté, que les couches archéologiques apparaissent nettement et qu'un simple coup d'oeil soit suffisant pour juger de la présence ou de l'absence d'un niveau paléolithique. Cela est peut ètre vrai pour les abris sous roche. II en va tout autrement en plein air tout au moins pour les gisements de la vallée de l'Isle. Les sols acides et lessivés ont perdu leur faune, leur charbon, leur cendre et tous les éléments qui pourraient les mettre en évidence. II ne reste plus que les silex et ces derniers apparaissent trés mal. La station de Solvieux en témoigne.

Solvieux est une vaste station de plein air, la plus riche à ce jour de tout le sud ouest de la France. Elle comporte en effet au dessus d'un moustérien indéterminé 13 niveaux du Paléolithique supérieur (Beauronnien, Aurignacien, Solutréen, Magdalénien) quelques vestiges d'un Néolithique récent et une occupation du Bronze final (C. Chevillot et J. Gaussen 1978; J. Gaussen 1980; J. Sackett à paraitre).

De nombreuses coupes de terrain y ont été éffectuées. La première est pratiquée tout au début des fouilles et examinée par des préhistoriens géologues de formation. Deux niveaux magdaléniens apparaissent mais le Périgordien sous jacent bien que intéréssé par la coupe, ne se remarque pas. II ne sera repéré que l'anné suivante aprés les pluies de l'hiver.

Une tranchée sondage est creusée dans la partie haute de la station jusqu'au sol stérile. Les coupes sont èxaminées par le Professeur J. Sackett et son équipe de fouilleurs puis dans les semaines suivantes par divers préhistoriens et par des géologues spécialisés dans la sédimentologie du Quaternaire. Les niveaux magdaléniens et périgordiens sont immédiatament reconnus mais l'Aurignacien et le Beauronien ne sont pas visibles. Ils n'apparaissent que quelques mois plus tard. Bien mieux, l'emplacement d'une industrie solutréenne incontestable ne pourra jamais ètre repérèe avec précision (un secteur restreint mis à part) malgré les examens aussi minutieux que fréquents. 
Par la suite, de nouvelles coupes de terrain apparaissent. Les préhistoriens n'en sont pas les auteurs. II s'agit de travaux en déblais, préliminaires à la construction d'une route que les archéologues, éternels gèneurs, ont retardé pendant plus de dix ans. La direction des antiquités d'Aquitaine me demande une ultime surveillance.

La tranchée, en déblai, doit entamer la partie superficielle de la couche moustérienne. Je la retrouve non sans quelques difficultés mais si je n'en avais pas connu l'emplacement précis je ne l'aurai pas remarquée. La tranchée entame également la partie marginale de l'un des niveaux périgordiens et, peut ètre aussi, le Bronze final. Je n'ai pas pu les repérer sur la coupe, et pourtant, j'ai la quasi certitude que l'occupation préhistorique s'étendait jusque la.

Tout ceci peut étonner et seuls peuvent le comprendre ceux qui sont habitués aux recherches et aux fouilles en plein air. Celles ci ne peuvent ètre comparées à celles qui ont lieu sous les abris rocheux ou dans les entrées de grottes. Les conditions sont essentiellement différentes. A la richesse et à l'entassement des uns s'oppose la pauvreté fréquente et l'éparpillement des autres.

La densité de quelques couches de Solvieux est exceptionnelle et ne se retrouve sur aucun autre gisement de plein air de la vallée de l'Isle. II y a bien par endroits quelques concentrations mais comme leur superficie n'est jamais très importante elles ne sont pas forcément rencontrées par une coupe de terrain dont le tracé n'a rien à voir avec la recherche archéologique. Elles le sont encore moins avec les sondages ponctuels. $\mathrm{Si}$ l'on imagine par exemple un terrain de un hectare contenant une concentration de pièces de deux à trois mètres carrés de superficie, les lois mathématiques de probabilité veulent qu'il faudra plusieurs milliers de sondages avant d'aboutir à sa découverte. Je viens de parler de concentrations mais lorsque la densité de l'industrie est peu importante les chances de découvertes s'amenuisent de façon considérable. Imaginons par exemple une couche riche de dix piéces de deux centimètres de longueur par mètre carré. II faudra une coupe de cinq mètres de longueur avant que n'apparaisse un objet. Si l'on ajoute à cela que bien des éléments sont arrachés et disparaissent dans les déblais ce n'est plus cinq, mais quinze à vingt mètres qui seront nécéssaires pour aboutir à une trouvaille. Ajoutons encore que bien de ces gisements n'étant pas très étendus il est facile de comprendre comment une station préhistorique de plein air peut passer inapercue malgré l'attention et la bonne volonté du chercheur. 
Heureusement il n'en est pas toujours ainsi et en certains cas quelques indices viennent au secours du prospecteur. Parfois les vestiges archéologiques sont contenus à l'intérieur d'une couche géologique bien individualisée et qui attire une attention particulière dans la mesure ou l'on sait qu'elle est contemporaine d'une civilisation bien précise. Dans d'autres cas les conditions physico-chimiques des terrains encaissants ont facilité une bonne conservation des restes humains. Dans les sols à $\mathrm{PH}$ neutre ou alcalin la faune n'a pas toujours été détruite et sa présence est le bon indicateur d'une occupation anthropique. Dans les couches rapidement enfouies le lessivage n'a pas eu le temps de faire disparaitre les débris charbonneux, les cendres et les matières colorantes si abondantes en certains cas.

Ces conditions favorables ne sont pas très fréquentes. Elles existent dans certaines régions privilégiées du bassin parisien, de la Rhénanie, du nord de l'Allemagne et de quelques pays plus lointains, mais le sud ouest de la France en est presque complètement dépourvu. Comment donc faut il rechercher et si possible découvrir les stations paléolithiques de plein air?

II n'y a pas de règles précises en la matière. II faut se rappeller tout d'abord que le hasard et la chance sont les facteurs essentiels de toute découverte. Mais ce hasard et cette chance doivent ètre cultivés. Cela exige une attention soutenue constituée de recherches les plus diverses et dominant tout cela: la conviction formelle que tout n'est pas encore connu. 


\section{BIBLIOGRAPHIE}

Chevillot, C. et Gaussen, J., 1978: "Une cachette du Bronze final III B sur le site préhistorique de Solvieux (St. Louis en l'Isle, Dordogne)". B.S.H.A.P., tome 105, n. ${ }^{\circ}$ 3, pag. 196-223, 16 fig.

Gaussen, J., 1980: Le Paléolithique supérieur de plein air en Périgord (secteur Mussidan St. Astier - moyenne vallée de l'Isle). XIVème suppl. à Gallia Préhistoire, édit du C.N.R.S. 305 pages, 127 fig., VIII pl.

Gaussen, J. et Moissat, J. C., 1989: “La station du Burin». A.D.R.A.P., sous presse.

Peyrille, L., 1948: “Note de séance». B.S.H.A.P., tome 75, 1 ére livraison, page 15.

Peyrille, L. et Blanc, S., 1952: "Le gisement de Beaufort près de St Louis en l'Isle (Dordogne)». Bull. de la soc. prèh. des amis des Eyzies, 2.

Peyrony, D., 1944: Le Périgord préhistorique - Essai de géographie humaine suivi des listes des stations, gisements et monuments divers avec leur bibliographie. Publ. de la S.H.A.P., 82 pag.

SACKETT, J.: "Solvieux et les fouilles de l'Université de Californie". Á paraitre. 
